\title{
The expression of phospho-AKT1 and phospho-MTOR is associated with a favorable prognosis independent of PTEN expression in intrahepatic cholangiocarcinomas
}

Dakeun Lee ${ }^{1,6}$, In-Gu Do ${ }^{2,6}$, Kyusam Choi ${ }^{2}$, Chang Ohk Sung ${ }^{1}$, Kee-Taek Jang ${ }^{1}$, Dongwook Choi ${ }^{3}$, Jin Seok $\mathrm{Heo}^{3}$, Seoung Ho Choi ${ }^{3}$, Jongmin Kim ${ }^{4}$, Jin Young Park ${ }^{4}$, Hyung Jin Cha ${ }^{5}$, Jae-Won Joh ${ }^{3}$, Kwan Yong Choi ${ }^{5}$ and Dae Shick Kim ${ }^{1}$

${ }^{1}$ Department of Pathology, Samsung Medical Center, Sungkyunkwan University School of Medicine, Seoul, Korea; ${ }^{2}$ Experimental Pathology Center, Samsung Cancer Research Institute, Samsung Medical Center, Seoul, Korea; ${ }^{3}$ Department of Surgery, Samsung Medical Center, Sungkyunkwan University School of Medicine, Seoul, Korea; ${ }^{4}$ CbsBioscience, Yuseong-gu, Daejeon, Korea and ${ }^{5}$ Department of Life Science, Pohang University of Science and Technology, Pohang, Korea

\begin{abstract}
AKT1 signaling pathway is important for the regulation of protein synthesis and cell survival with implications in carcinogenesis. In this study, we explored the prognostic significance of AKT1 pathway in intrahepatic cholangiocarcinomas. We investigated the status of phosphatase and tensin homolog deleted on chromosome 10 (PTEN), phosphorylated (p) AKT1 (p-AKT1), p-mammalian target of rapamycin (p-MTOR), p-p70 ribosomal protein S6 kinase (p-RPS6KB2) and p-eukaryotic initiation factor 4E-binding protein-1 (p-EIF4EBP1) in 101 intrahepatic cholangiocarcinomas by immunohistochemistry. Western blot analysis was performed to verify the expression levels of p-AKT1 and p-MTOR. The relationship of protein expression with clinicopathological data and the correlations of protein expression levels were explored. The overexpression of p-AKT1, p-MTOR, and PTEN was associated with a better survival in patients with intrahepatic cholangiocarcinoma $(P=0.0137$, 0.0194 , and 0.0337 , respectively). In a multivariate analysis, PTEN was an independent prognostic factor, and p-AKT1 showed tendency $(P=0.032$ and 0.051 , respectively). The overexpression of $p$-MTOR was correlated with well-to-moderately differentiated tumors $(P<0.001)$ and tumors without metastasis $(P=0.046)$. Expression levels of the AKT1 signaling pathway proteins in this study showed positive correlations with each other, except for PTEN. Aberrant expressions of p-AKT1 and p-MTOR in intrahepatic cholangiocarcinoma were associated with a favorable prognosis, possibly in a PTEN-independent manner. Our results indicate that dysregulation of the AKT1 pathway may have an important role in the development of intrahepatic cholangiocarcinoma, but not necessarily in the progression of the disease.

Modern Pathology (2012) 25, 131-139; doi:10.1038/modpathol.2011.133; published online 26 August 2011
\end{abstract}

Keywords: AKT1; intrahepatic cholangiocarcinoma; MTOR; prognosis; PTEN

Cholangiocarcinoma is a rare neoplasm in the developed world, and this tumor arises from the

Correspondence: Dr DS Kim, MD, PhD, Department of Pathology, Samsung Medical Center, Sungkyunkwan University School of Medicine, 50 Irwon-dong, Gangnam-gu, Seoul 135-710, Republic of Korea.

E-mail: oncorkim@skku.edu

${ }^{6}$ These authors contributed equally to this paper.

Received 15 February 2011; revised 13 July 2011; accepted 18 July 2011; published online 26 August 2011 epithelial cells of the bile ducts. Anatomically cholangiocarcinomas are classified as intrahepatic cholangiocarcinomas, hilar cholangiocarcinomas, and distal extrahepatic bile duct cancers. Intrahepatic cholangiocarcinoma usually refers to a tumor originating within the second bifurcation of hepatic ducts. The incidence of intrahepatic cholangiocarcinoma is even lower as it accounts for less than $10 \%$ of all cholangiocarcinomas, ${ }^{1}$ although some recent studies have showed its increasing incidence in the West. ${ }^{2,3}$ Complete surgical resection 
is the only treatment that offers the possibility of long-term survival in patients with intrahepatic cholangiocarcinoma. However, the number of candidates who can undergo curative surgery is limited, because a significant proportion of patients present with advanced disease. ${ }^{2}$ Even for patients who undergo surgical resection, the 5-year survival rate is still only $22-36 \% .^{4}$ At present, little is known about the molecular carcinogenesis of intrahepatic cholangiocarcinoma. A better knowledge of the molecular pathways involved in the development and progression of this disease may help devise new treatment strategies to improve the clinical outcome of patients with intrahepatic cholangiocarcinoma.

AKT1 (also known as protein kinase B) is a serine/ threonine protein kinase and has a central role in the regulation of diverse biological functions. Phosphatidylinositol 3-kinase (PIK3CA), an upstream regulator of $\mathrm{AKT} 1$, is activated by receptor tyrosine kinases such as epidermal growth factor receptor or insulin-like growth factor 1 receptor, and it catalyzes the conversion of phosphatidylinositol-4,5-bisphosphate (PIP2) to phosphatidylinositol-3,4,5-triphosphate (PIP3). PIP3 binds to AKT1, leading to the membrane recruitment of AKT1 and phosphorylation of AKT1..$^{5}$ Activated AKT1 phosphorylates, activates, or inhibits many downstream targets to regulate various cellular functions, including cell metabolism, protein synthesis, cell survival, inhibition of apoptosis, and cell cycle progression. ${ }^{5,6}$ The aberrant expression or deregulation of AKT1 has been observed in many different types of tumors, and it is thought to be an important step for tumorigenesis or disease progression in these tumors. ${ }^{7-9}$ On the other hand, the phosphatase and tensin homolog deleted on chromosome 10 (PTEN) was first discovered as a tumor suppressor on human chromosome 10q23. PTEN has a lipid phosphatase activity, and it hydrolyzes the 3-phosphate on PIP3 to generate PIP2; therefore, PTEN negatively regulates AKT $1 .{ }^{10}$ Specifically, the loss or mutation of PTEN leads to activation of AKT1, which in turn promotes the anti-apoptotic and pro-cell cycle entry pathways, which are believed to be essential in tumorigenesis. A series of studies have shown that PTEN is frequently mutated or lost in many human malignancies, including glioblastomas, breast cancer, lung cancer, colon cancer, and melanomas. ${ }^{11-13}$ In addition, decreased PTEN expression has been correlated with progression of solid cancers. ${ }^{14,15}$

Mammalian target of rapamycin (MTOR) is a member of the PIK3CA-related kinase family, and it is the key downstream signaling molecule of AKT1. Activated MTOR supports cell growth, cell survival, and protein synthesis, largely by phosphorylating two downstream targets: p70 ribosomal protein S6 kinase (RPS6KB2) and eukaryotic initiation factor $4 \mathrm{E}$ (eIF4E)-binding protein-1 (EIF4EBP1). ${ }^{16}$ Activated p70S6K subsequently phosphorylates specific ribosomal protein S6, and phosphorylated S6, in turn, promotes protein translation and cell growth. Hypophosphorylated EIF4EBP1 binds to, and thereby inactivates the capbinding protein eIF4E, whereas phosphorylation of EIF4EBP1 results in release of eIF4E, which then binds to the cap structure of mRNA to initiate protein translation. ${ }^{17}$ As all of these proteins are regulators of protein synthesis, they can act as protooncogenes, and in fact, they are frequently activated in human malignancies. ${ }^{18,19}$ Accordingly, the suppression of MTOR activity could inhibit cell growth, eliciting an anti-tumor effect. Inhibitors of proteins that are involved in MTOR signaling, such as rapamycin (sirolimus) and its analogs CCI-779 (temsirolimus), have recently been under active preclinical or clinical investigations for treating cancers, and they have been suggested as potential therapeutic agents. ${ }^{20}$

Although the AKT1 signaling pathway has been under active investigation in a variety of cancers, there has been limited in vivo data regarding the activity or the prognostic value of the signaling proteins of AKT1 pathway in intrahepatic cholangiocarcinoma. Further, the coexpression of these proteins in intrahepatic cholangiocarcinoma has not yet been described. To better understand the role of AKT1 signaling pathway in the tumorigenesis and progression of intrahepatic cholangiocaricnoma, we investigated the statuses of the proteins in this pathway, and their correlations with the clinicopathological data in patients with intrahepatic cholangiocarcinoma. Survival analysis was performed to assess the prognostic relevance of these biomarkers. Furthermore, we sought to explore the correlations between the expression levels of the signaling proteins, and we investigated whether the conventional model of the PTEN-AKT1-MTORRPS6KB2/EIF4EBP1 pathway is valid in this type of tumor. Thus, this study will have important implications for further elucidating the molecular mechanisms, and finding novel therapeutic targets in this rare subtype of cholangiocarcinomas.

\section{Patients and methods}

\section{Patients and Tumor Samples}

The study subjects included 101 patients with intrahepatic cholangiocarcinoma, who were surgically treated at Samsung Medical Center (Seoul) from July 1996 to December 2007. All the tumor samples were from the primary cholangiocarcinoma at the time of surgical resection. None of the patients underwent chemotherapy or radiotherapy before surgery. Post-operative adjuvant treatments were performed in a subset of patients as follows: chemotherapy in 10 , radiotherapy in 5 , and both modalities in 12 patients. The cholangiocarcinomas that had their epicenter only within the hepatic parenchyma were included, whereas carcinomas of the hilar or perihilar bile ducts (Klatskin tumors) 
and carcinomas of the mid to distal bile duct were excluded. Carcinomas arising in the gallbladder or extrahepatic bile duct, with extension to the intrahepatic bile duct, were also excluded in this study. All the tumors were classified according to the World Health Organization classification. ${ }^{21}$ The medical records were reviewed to obtain data that included the age and gender of the patients, the survival time, and the survival status. The histopathologic data such as the tumor location, size, and histological type were obtained from the pathology reports. In this study, we used pT classification by AJCC $6^{\text {th }}$ edition. This study received appropriate human protection approval from the Institutional Review Board of Samsung Medical Center.

\section{Immunohistochemistry}

Immunohistochemical (IHC) studies were carried out on formalin-fixed, paraffin-embedded, $4-\mu \mathrm{m}$ thick tissue sections. The primary antibodies used were rabbit monoclonal anti-human PTEN (138G6, 1:200 dilution, Cell Signaling Technology, Beverly, MA, USA), phosphorylated (p) AKT1 (p-AKT1) (D9E, 1:20 dilution, Cell Signaling Technology), p-MTOR; (49F9, 1:50 dilution, Cell Signaling Technology), p-RPS6KB2 (1A5, 1:500 dilution, Cell Signaling Technology), and p-EIF4EBP1 (236B4, 1:100 dilution, Cell Signaling Technology). The tissue sections were deparaffinized three times in xylene for a total of $15 \mathrm{~min}$, and they were subsequently rehydrated. Immunostaining for PTEN, p-MTOR, p-RPS6KB2, and p-EIF4EBP1 was performed using a Bond-max autoimmunostainer (Leica Biosystem, Melbourne, VIC, Australia) with ER1 or ER2 retrieval buffers, and a Bond Polymer refine detection system DS9800 (Vision Biosystems, Melbourne, VIC, Australia). For the immunostaining for $\mathrm{p}-\mathrm{AKT} 1$, the slides were autoclaved for $1 \mathrm{~min}$ at $110^{\circ} \mathrm{C}$ in citrate buffer ( $\mathrm{pH}$ 6.0). After the endogenous peroxidase activity was blocked with $3 \%$ hydrogen peroxidase for $10 \mathrm{~min}$, the primary antibody incubation was carried out overnight at $4{ }^{\circ} \mathrm{C}$. The antigen-antibody reaction was detected using the DAKO REAL Envision Detection system,
Peroxidase/DAB K5007 (DAKO, Glostrup, Denmark). Counter-staining was performed with Mayer's hematoxylin, and then the slides were examined by light microscopy. Appropriate positive controls were selected according to the manufacturer's recommendations. The slides processed without the primary antibodies were used as negative controls.

\section{Interpretation of IHC Staining}

The expressions of PTEN, p-AKT1, p-MTOR, p-RPS6KB2, and p-EIF4EBP1 were examined by two pathologists (DL and COS), who were blinded to the clinical data of the patients. The pathologists inspected the complete tumor sections at $\times 40$ and $\times 200$ magnifications. The discordant cases were reviewed under a multi-headed microscope to achieve a consensus. In this study, the tumor cells showing cytoplasmic staining with or without the nucleus or cell membrane staining were judged to be positive for PTEN, p-AKT1, p-MTOR, and p-EIF4EBP1, whereas nucleus staining was considered positive for p-RPS6KB2. The staining intensity was determined as 0 (absent), 1 (weak), 2 (moderate), and 3 (strong). The photomicrographs of the biomarkers tested strong positive in tumor tissue are depicted in Figure 1. On the other hand, PTEN, p-AKT1, and p-EIF4EBP1 showed weak cytoplasmic staining in normal bile ducts in some cases. p-MTOR showed strong cytoplasmic reactivity in normal bile ducts as previously described, ${ }^{22}$ whereas p-RPS6KB2 was not expressed in normal bile ducts. For comparison with the clinical variables and survival, the expression levels of these proteins were semiquantified using an IHC score calculated by multiplication of the staining intensity $(0 \sim 3)$ with the percentage of positive tumor cells $(0 \sim 100 \%)$. The ranges of IHC scores for each biomarker in tumor tissue are as follows: $0 \sim 200$ for PTEN (median 3), 0 140 for p-AKT1 (median 10), $0 \sim 285$ for p-MTOR (median 60), 0 297 for p-EIF4EBP1 (median 10), and 0 120 for p-RPS6KB2 (median 0) (Supplementary Figure S1). The cut-off values for each biomarker were set to best
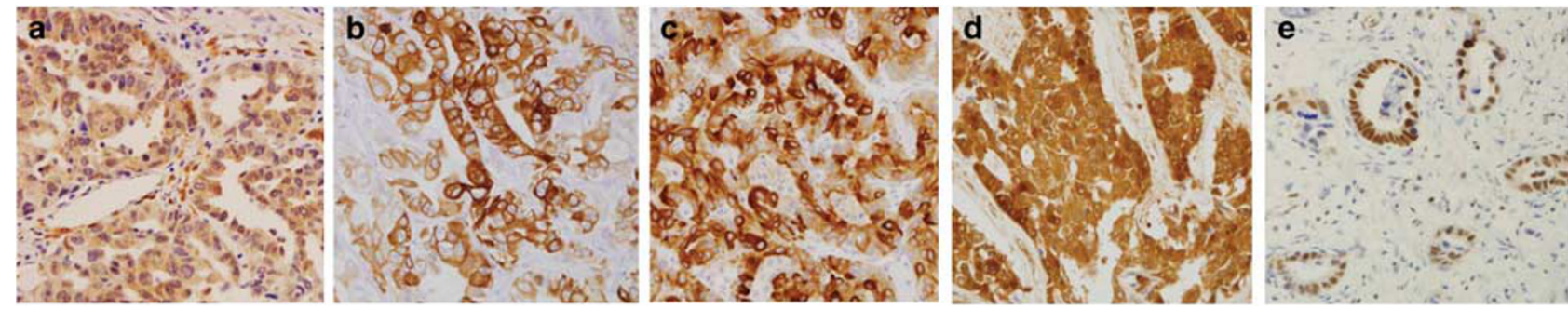

Figure 1 The photomicrographs of IHC staining that showed strong reactivity in this study. (a) PTEN, (b) p-AKT1, (c) p-MTOR, and (d) p-EIF4EBP1 show strong cytoplasmic staining of the tumor cells, and some positive cells stained for p-AKT1 and p-MTOR also exhibit membranous staining in these examples. On the other hand, (e) p-RPS6KB2 demonstrates strong nuclear reactivity in the intrahepatic cholangiocarcinoma specimens. 
discriminate the patients' survival statistically. The cut-off IHC scores that were considered positive are as follows: > 15 for PTEN, p-AKT1, and p-RPS6KB2, and $>50$ for $\mathrm{p}$-MTOR and p-EIF4EBP1. The cases with IHC scores below the cut-off values were considered negative.

\section{Western Blot Analysis}

To verify the correlations of IHC scores with expression levels of p-AKT1 and p-MTOR in the intrahepatic cholangiocarcinoma, western blot analysis was performed for the eight cases of intrahepatic cholangiocarcinoma for which fresh-frozen tumor tissues were available. Frozen tissue examination confirmed that $70-80 \%$ of the specimens were tumor tissue. The frozen tissues were homogenized in tissue lysis buffer $(20 \mathrm{mM}$ HEPES (pH 7.4), $75 \mathrm{mM} \mathrm{NaCl}, 2.5 \mathrm{mM} \mathrm{MgCl} 2,0.1 \mathrm{mM}$ EDTA, $0.05 \%$ (v/v) Triton X-100, $20 \mathrm{mM}$ betaglycerophosphate, $1 \mathrm{mM}$ Na3VO4, $10 \mathrm{mM} \mathrm{NaF}$, $0.5 \mathrm{mM}$ DTT, and the protease inhibitor complete Mini (Roche, Penzberg, Germany)). The samples were incubated for $15 \mathrm{~min}$ on ice, and then, they were centrifuged at $14000 \mathrm{~g}$ at $4{ }^{\circ} \mathrm{C}$ for $30 \mathrm{~min}$. The samples containing $15 \mu \mathrm{g}$ of protein was separated on $10 \%$ SDS-polyacrylamide gel, and then, the proteins were transferred to nitrocellulose membranes. These membranes were blocked in Trisbuffered saline containing 5\% skim milk and $0.05 \%$ Tween-20 (TBST) for $1 \mathrm{~h}$ at room temperature, before incubation with the primary antibody overnight at $4{ }^{\circ} \mathrm{C}$. Western blotting was conducted using a 1:1500 dilution of antibody against p-AKT1 (Cell Signaling Technology, Beverly, MA) and a 1:1000 dilution of antibody against p-MTOR (Cell Signaling Technology). Peroxidase-conjugated goat antimouse IgG diluted 1:5000 was used as a secondary antibody. Peroxidase was visualized via an enhanced chemiluminescence assay (Amersham, Piscataway, NJ, USA). The images of the gels were scanned using a Gel Documentation System (Gel Doc 1000, Bio-Rad, Hercules, CA, USA), and the relative densities were analyzed using a Multianalyst fingerprinting program (version 1.1, Bio-Rad).

\section{Statistical Analysis}

The statistical analyses were done using SPSS for Windows (version 12.0, SPSS Inc, Chicago, IL, USA). The associations between the expression of each protein and the clinicopathological variables were examined by Pearson's $\chi^{2}$-test or Fisher's exact test. Spearman's rank test was used to determine the relationships between the expression levels of any two proteins. The survival rates were estimated using the Kaplan-Meier method, and the survival curves between groups stratified by clinicopathological parameters and protein expression levels were compared by the log-rank test. Multivariate analysis for survival was done using a Cox proportional hazards regression model. A $P$-value of less than 0.05 was considered statistically significant. All the reported $P$-values are two-sided.

\section{Results}

\section{Clinicopathological Characteristics}

The ages of the patients ranged from 13-76 years (median age: 59 years). Sixty patients were men and 41 were women. The tumor sizes ranged from $1-15.8 \mathrm{~cm}$ (median: $4.5 \mathrm{~cm}$ ). Most of the cases (92 out of 101) were histologically diagnosed as adenocarcinoma, whereas others included mucinous carcinoma (three cases), adenosquamous carcinoma (one case), sarcomatous carcinoma (three cases), and bile duct cystadenocarcinoma (two cases). Only for 92 cases of adenocarcinomas, tumor differentiation was assessed. Of these, 64 cases were well-to-moderately differentiated, whereas 28 cases were poorly differentiated. The patients' follow-up time ranged from 0.6-158.2 months, and the median survival at the last follow-up was 21.8 months.

\section{Relationships between the Clinicopathological Variables and the Protein Expressions}

The survival rates according to each clinicopathological parameter assessed by Kaplan-Meier method are summarized in Table 1. The significant prognostic factors for poor survival were the presence of satellite nodule(s) $(P=0.0013)$, poorly differentiated tumor $(P=0.0257)$, presence of vascular invasion $(P=0.0029)$, perineural invasion $(P=0.0034)$, and lymph node metastasis $(P<0.0001)$. The pT classification also had an impact on the prognosis $(P=0.0181)$. Gender, age, and the resection margin status were not significantly correlated with the patients' survival. The size of tumor only showed a trend $(P=0.0524)$. Pearson's $\chi^{2}$-test or Fisher's exact test was used to investigate the relationships between the clinical variables and the expressions of the analyzed pathway proteins (Supplementary Table S1). The expression of p-MTOR was more frequently observed in the well-to-moderately differentiated tumors $(P<0.001)$ and in the tumors without lymph node metastasis $(P=0.046)$.

\section{Status of the Expressions of Signaling Proteins and their Relationships with Survival}

Positive reactivity for PTEN, p-AKT1, p-MTOR, p-RPS6KB2, and p-EIF4EBP1 was found in $33.7 \%$. (34 of 101 cases), $33.7 \%$ (34 cases), $53.5 \%$ (54 cases), $22.8 \%$ (23 cases), and $23.8 \%$ (24 cases), respectively, of all the intrahepatic cholangiocarcinoma cases. The expression of p-AKT1 and p-MTOR was further confirmed by western blot analysis in eight representative patients whose fresh-frozen tissues were 
Table 1 Overall survival rates of the patients with intrahepatic cholangiocarcinoma according to the clinicopathologic variables

\begin{tabular}{|c|c|c|c|c|c|}
\hline & No. & $\begin{array}{c}\text { 1-year } \\
(\%)\end{array}$ & $\begin{array}{c}\text { 3-year } \\
(\%)\end{array}$ & $\begin{array}{c}5 \text {-year } \\
(\%)\end{array}$ & $\mathrm{P}$-value \\
\hline \multicolumn{6}{|l|}{ Gender } \\
\hline Male & 60 & 58.33 & 39.36 & 31.53 & \multirow[t]{2}{*}{0.62} \\
\hline Female & 41 & 75.61 & 43.62 & 34.98 & \\
\hline \multicolumn{6}{|l|}{ Age } \\
\hline$<65$ & 53 & 66.04 & 40.82 & 31.02 & \multirow[t]{2}{*}{0.92} \\
\hline$\geqslant 65$ & 48 & 64.58 & 41.16 & 35.84 & \\
\hline \multicolumn{6}{|l|}{ Satellite nodule(s) } \\
\hline Present & 15 & 33.33 & 13.33 & 13.33 & \multirow[t]{2}{*}{$0.0013^{*}$} \\
\hline Absent & 86 & 70.93 & 45.77 & 36.32 & \\
\hline \multicolumn{6}{|l|}{ Size $(\mathrm{cm})$} \\
\hline$\leqslant 5$ & 63 & 73.02 & 44.89 & 39.01 & \multirow[t]{2}{*}{0.0524} \\
\hline$>5$ & 38 & 52.63 & 31.58 & 21.65 & \\
\hline \multicolumn{6}{|l|}{ Differentiation } \\
\hline Well/moderate & 64 & 67.19 & 45.12 & 35.71 & \multirow[t]{2}{*}{$0.0257^{*}$} \\
\hline Poor & 28 & 53.57 & 21.43 & 16.07 & \\
\hline \multicolumn{6}{|l|}{ Vascular invasion } \\
\hline Present & 42 & 47.62 & 25.54 & 19.15 & \multirow[t]{2}{*}{$0.0029^{*}$} \\
\hline Absent & 59 & 77.97 & 51.83 & 40.9 & \\
\hline \multicolumn{6}{|c|}{ Perineural invasion } \\
\hline Present & 31 & 51.61 & 21.77 & 12.1 & \multirow[t]{2}{*}{$0.0034^{*}$} \\
\hline Absent & 70 & 71.43 & 49.32 & 41.75 & \\
\hline \multicolumn{6}{|l|}{$L N$ metastasis } \\
\hline Present & 27 & 33.33 & 18.52 & 12.35 & \multirow[t]{2}{*}{$<0.0001^{*}$} \\
\hline Absent & 74 & 77.03 & 49.23 & 40.04 & \\
\hline \multicolumn{6}{|l|}{ Resection margin } \\
\hline Involved & 7 & 85.71 & 28.57 & 28.57 & \multirow[t]{2}{*}{0.76} \\
\hline Clear & 94 & 63.83 & 42 & 33.33 & \\
\hline \multicolumn{6}{|l|}{ pT classification } \\
\hline 1 & 52 & 76.92 & 54.95 & 42.28 & \multirow[t]{4}{*}{$0.0181^{*}$} \\
\hline 2 & 32 & 62.5 & 26.52 & 19.89 & \\
\hline 3 & 15 & 33.33 & 26.67 & 26.67 & \\
\hline 4 & 2 & 50 & 0 & 0 & \\
\hline
\end{tabular}

* Significant at the level of $P<0.05$.

Survival rates were estimated using Kaplan-Meier method and the $P$-value was drawn by log-rank test.

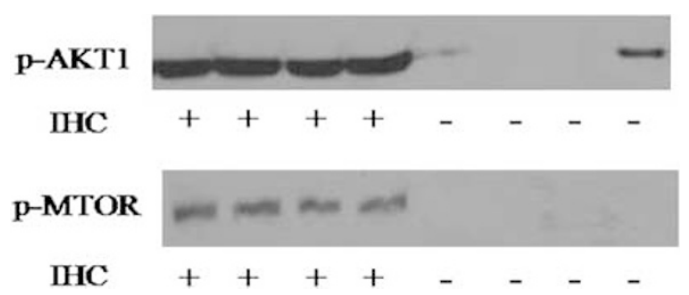

Figure 2 Representative results of the western blot analysis of the intrahepatic cholangiocarcinoma specimens. Eight patients with positive $(+)$ or negative $(-)$ p-AKT1 or p-MTOR expression as assessed by immunohistochemistry were analyzed by western blot analysis to validate these antibodies.

available, and there was a tight correlation between the results of the immunohistochemistry and the western blot analysis (Figure 2). The 5-year overall survival rate was significantly higher for the patients
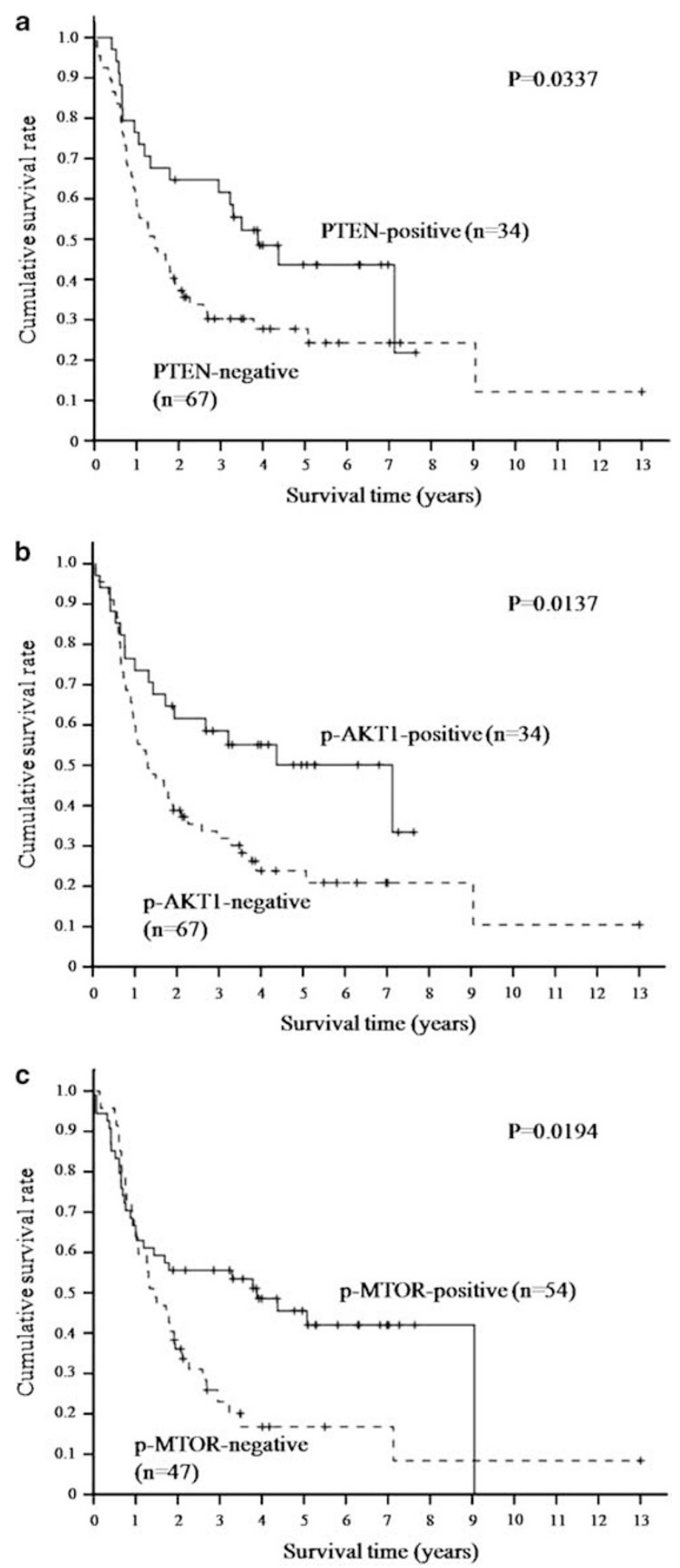

Figure 3 Kaplan-Meier survival analysis of the intrahepatic cholangiocarcinomas for comparing the overall survival for (a) PTEN, (b) p-AKT1, and (c) p-MTOR.

whose tumors overexpressed PTEN $(P=0.0337)$, p-AKT1 $(P=0.0137)$, and p-MTOR $(P=0.0194$; Figure 3). Patients with tumors that were positive for p-RPS6KB2 and p-EIF4EBP1 showed better 
Table 2 Correlations between the expressions of PTEN, p-AKT1, p-MTOR, p-RPS6KB2, and p-EIF4EBP1

\begin{tabular}{|c|c|c|c|c|c|}
\hline & $\begin{array}{c}\text { PTEN } \\
\text { (P-value) }\end{array}$ & $\begin{array}{c}p-A K T 1 \\
\text { (P-value) }\end{array}$ & $\begin{array}{l}\text { p-MTOR } \\
\text { (P-value) }\end{array}$ & $\begin{array}{c}p \text {-RPS6KB2 } \\
(\mathrm{P} \text {-value) }\end{array}$ & $\begin{array}{c}p \text {-EIF4EBP1 } \\
\text { (P-value) }\end{array}$ \\
\hline \multicolumn{6}{|l|}{ PTEN } \\
\hline p-AKT1 & $0.116(\rho=0.158)$ & & & & \\
\hline p-MTOR & $0.238(\rho=0.119)$ & $0.042 *(\rho=0.203)$ & & & \\
\hline p-RPS6KB2 & $0.533(\rho=0.063)$ & $0.033 *(\rho=0.213)$ & $0.025^{*}(\rho=0.223)$ & & \\
\hline p-EIF4EBP1 & $0.015^{*}(\rho=0.242)$ & $<0.001 *(\rho=0.390)$ & $0.015^{*}(\rho=0.241)$ & $<0.001 *(\rho=0.362)$ & \\
\hline
\end{tabular}

Abbreviations: PTEN, phosphatase and tensin homolog deleted on chromosome 10; p-AKT1, phosphorylated AKT1; p-MTOR, phosphorylated mammalian target of rapamycin; p-RPS6KB2, phosphorylated p70 ribosomal protein S6 kinase; p-EIF4EBP1, phosphorylated eukaryotic initiation factor 4E-binding protein-1; $\rho$, Spearman's coefficient.

* Significant at the level of $P<0.05$.

overall survival rates, but the differences were not statistically significant $(P=0.3164$ and 0.097 , respectively; Supplementary Table S2).

\section{Correlations between the Expressions of the Signaling Proteins}

The correlations between the expression levels of the semi-quantitatively assessed biomarkers were explored using Spearman's rank test (Table 2). On this analysis, significant correlations were found in many pairs of proteins analyzed, and the Spearman's coefficients $(\rho)$ were all positive values. The expression of p-AKT1 was correlated with the expression of p-MTOR $(P=0.042)$. The expression of p-EIF4EBP1 was strongly correlated with the expressions of PTEN $(P=0.015), \quad$-AKT1 $(P<0.001)$, p-MTOR $(P=0.015)$, and p-RPS6KB2 $(P<0.001)$. The expression of p-RPS6KB2 was also positively correlated with the expressions of p-AKT1 and p-MTOR $(P=0.033$ and 0.025 , respectively). On the other hand, no correlation was observed between the expression of PTEN and the expressions of the other proteins, except for p-EIF4EBP1.

\section{Multivariate Analysis of the Clinicopathological Factors for Survival}

Multivariate analysis was done with a Cox regression model, and we included only the clinical variables and biomarkers that showed significance on the univariate analysis (Table 3). We found expression of PTEN $(P=0.032)$ was an independent favorable prognostic factor, whereas the presence of vascular invasion $(P=0.017)$, perineural invasion $(P=0.020)$, and $\mathrm{LN}$ metastasis $(P<0.001)$ were independent prognostic factors that predicted poor survival. The expression of p-AKT1 showed a tendency, but did not attain statistical significance in the multivariate analysis $(P=0.051)$.

\section{Discussion}

By evaluating expression of multiple components of the AKT1 pathway in a large number of intrahepatic cholangiocarcinomas using immunohistochemistry,
Table 3 Multivariate analysis by the Cox proportional hazard regression model

\begin{tabular}{lccc}
\hline & $\begin{array}{c}\text { Hazard } \\
\text { ratio }\end{array}$ & $95 \%$ CI & P-value \\
& & & \\
\hline Poorly differentiated tumor & 1.598 & $0.880-2.901$ & 0.124 \\
Presence of vascular invasion & 1.963 & $1.127-3.417$ & $0.017^{*}$ \\
Presence of perineural invasion & 1.867 & $1.105-3.154$ & $0.020^{*}$ \\
Presence of satellite nodule(s) & 1.626 & $0.771-3.426$ & 0.201 \\
Presence of LN metastasis & 4.135 & $2.240-7.636$ & $<0.001^{*}$ \\
Expression of PTEN & 0.523 & $0.290-0.946$ & $0.032^{*}$ \\
Expression of p-AKT1 & 0.549 & $0.301-1.003$ & 0.051 \\
Expression of p-MTOR & 1.064 & $0.609-1.859$ & 0.827 \\
\hline
\end{tabular}

Abbreviations: PTEN, phosphatase and tensin homolog deleted on chromosome 10; p-AKT1, phosphorylated AKT1; p-MTOR, phosphorylated mammalian target of rapamycin; CI, confidence interval. ${ }^{*}$ Significant at the level of $P<0.05$.

this study was able to show, for the first time, the interactions between various members of the AKT1 pathway including its downstream targets, p-RPS6KB2 and p-EIF4EBP1, and their prognostic significance in patients with intrahepatic cholangiocarcinoma. We found that the overexpression of PTEN was an independent favorable prognostic factor for intrahepatic cholangiocarcinoma, and that the overexpression of p-AKT1 and p-MTOR could also predict a favorable prognosis for patients with intrahepatic cholangiocarcinoma in the univariate analysis. In addition, AKT1 and its downstream pathway did not appear to be strongly influenced by PTEN in the intrahepatic cholangiocarcinoma.

AKT1 and its downstream proteins have been reported to be frequently activated in human cancers, and these activated forms were shown to be poor prognostic factors in several malignant tumors, including non-small cell lung cancer ${ }^{23}$ and breast cancer. ${ }^{24}$ Studies suggest that this may be the case for malignant tumors of the biliary tract as well. Herberger et $a l^{22}$ found that overall survival was significantly shorter for patients with p-MTORpositive tumors of the biliary tract, as compared with that of patients with p-MTOR-negative tumors. A recent study demonstrated that a low PTEN expression and decreased PTEN/p-AKT1 and PTEN/p-MTOR expression ratios in extrahepatic cholangiocarcinoma were poor prognostic factors, 
although there were no significant differences in survival rates based on the single protein expression status of p-AKT1 and p-MTOR. ${ }^{25}$ Moreover, an earlier study demonstrated that PIK3CA is an important mediator of Ras-induced radiation resistance in T24 (human bladder carcinoma) cells, ${ }^{26}$ and Tanno et $a l^{27}$ drew a similar conclusion that constitutively active AKT1 markedly decreased radiosensitivity in human bile duct cancer cells.

In contrast, in the present study, we found the overexpression of PTEN, p-AKT1, and p-MTOR could predict better survival in patients with intrahepatic cholangiocarcinoma. Similarly, patients with p-RPS6KB2- or p-EIF4EBP1-positive tumors exhibited better 5-year overall survival rates than patients with p-RPS6KB2- or p-EIF4EBP1negative tumors, although the differences were not statistically significant. These unexpected results are intriguing, as most of the previous studies, whether they dealt with cholangiocarcinoma or other cancers, considered overexpression of these biomarkers to be poor prognostic factors. To our knowledge, there are only two previous reports on human cholangiocarcinomas that are in line with our results. Javle et $a l^{28}$ studied 24 cases of cholangiocarcinoma using immunohistochemistry, and they revealed that AKT1 and p-AKT1 expressions were associated with an improved prognosis. However, that study did not provide the specific location of the tumor in the bile duct, and the number of cases was too small to draw meaningful conclusions. In another study, Schmitz et $a l^{29}$ showed that patients with strong positive p-AKT1 staining had higher 5-year survival rates among 59 patients with intrahepatic cholangiocarcinoma. However, they concluded that p-AKT1 expression was not a significant prognostic factor, because the differences did not reach statistical significance.

An important and well-known function of AKT1 signaling pathway is the maintenance of cell survival and inhibition of apoptosis. There is growing evidence, on the other hand, that this pathway is also involved in the promotion of cell death. Perez-Tenorio and $\mathrm{Stal}^{24}$ showed that p-AKT1 was strongly associated with a lower S-phase fraction, and Lawlor and Rotwein ${ }^{30}$ observed induction of the cell cycle inhibitor p21 by constitutively activated AKT1. In keeping with this, a recent study demonstrated that AKT1 sensitized cells to reactive oxygen species-mediated apoptosis by increasing intracellular levels of reactive oxygen species through an increase in oxygen consumption and the inhibition of FoxO transcription factors. ${ }^{31}$ In this study, rapamycin, a well-known MTOR inhibitor, rather sensitized cells to reactive oxygen speciesmediated cell death, because it paradoxically activated AKT1 via the inhibition of a negative feedback loop. In addition, Lu et $a l^{32}$ observed that stimulation of the Fas receptor induced rapid phosphorylation of AKT1, and a parallel increase in cell apoptosis in epidermal C141 cells.
It is questionable how AKT1 is assigned a dual role in both cell survival and cell death. There are some molecules that can trigger such opposing pathways. For example, well-described oncogenes such as Myc, Ras, and E2F1, which deliver strong mitogenic signals, have also been reported to cause cell death. ${ }^{33,34}$ In addition, insulin-like growth factor, which is a well-known mitogenic and antiapoptotic factor for many cells, was proven to significantly inhibit DNA synthesis and cell proliferation in a human lung adenocarcinoma cell line, possibly via the sustained activation of AKT1 and the induction of $\mathrm{p} 21 .^{35}$ Therefore, it is possible that the outcome of AKT1 activation may vary depending on the type of stimulus and the microenvironment, or activated AKT1 may direct signals in an organ (or tumor)-specific manner. For instance, BCL2, which is known to block apoptosis, promotes cell death when BCL2 interacts with nuclear orphan receptor Nur77. ${ }^{36}$ Nur77 binding induces a BCL2 conformational change, resulting in conversion of BCL2 as an opposing phenotype. In another study, insulin-like growth factor-1 and the adenovirusmediated overexpression of wild-type class I PIK3CA accelerates the accumulation of autophagic vacuoles and subsequent autophagic cell death during glucose deprivation. ${ }^{37}$ From this point of view, abnormally activated AKT signaling pathway by other than loss of PTEN in the current study could possibly change its downstream targets and result in the opposite outcome, or AKT1 might show a different action specifically in intrahepatic cholangiocarcinomas.

We observed that p-MTOR expression was associated with well-to-moderately differentiated tumors, and that p-MTOR was inversely correlated with metastatic disease. A positive correlation between p-MTOR and better survival was recently described in early-stage lung adenocarcinoma ${ }^{38}$ and gastric adenocarcinoma, ${ }^{39}$ and the association of MTOR and the intestinal type histology in gastric adenocarcinoma was also observed. Similar findings for p-AKT1 have also been reported. Shah et al ${ }^{40}$ reported that p-AKT1 was strongly correlated with well-differentiated tumors, and that p-AKT1 expression in non-small cell lung cancer was a favorable independent prognostic factor. In a study by Cappuzzo et $a{ }^{41}$ p-AKT1 was associated with bronchioloalceolar carcinoma, which is a well-differentiated form of adenocarcinoma, and p-AKT1 positivity was associated with a reduced risk of disease progression. Taken together, there are tumors with high p-AKT1 or p-MTOR expression that remain well differentiated, seldom metastasize, and thus are associated with a better survival. This suggests the possibility that the AKT1-MTOR pathway may have a pivotal role in the development of certain tumors rather than in disease progression. Lee et $a l^{42}$ supported this hypothesis by demonstrating that there was no difference in the p-AKT1 levels between primary non-small cell lung cancer and the corresponding metastatic node. 
Another notable finding in the present study is that the overexpression of PTEN was not associated with the overexpression of p-AKT1 and other downstream proteins, except for p-EIF4EBP1, in intrahepatic cholangiocarcinoma, whereas all the other proteins except for PTEN showed correlated expression levels with one another. This indicates that PTEN mutation or loss of PTEN expression does not necessarily lead to activation of the AKT1 pathway in intrahepatic cholangiocarcinoma. As there are numerous signaling molecules involved in the PTEN-AKT1 pathway with frequent crosstalk, it is not surprising that the expression of these signaling proteins could be closely correlated in some conditions, but not in others. A previous report described that a high level of p-AKT1 expression occurred independent of the presence of PTEN mutations in endometrial cancers. ${ }^{43}$ In a report regarding non-small cell lung cancer by Yoshizawa et al, ${ }^{23}$ there was a lack of association between p-AKT1 and p-MTOR expression. It is striking that Panigrahi et $a l^{44}$ found that the level of PTEN expression was even positively correlated with the level of the p-AKT1 expression in breast cancer. Moreover, RPS6KB2 and eIF4E can be activated by pathways that are independent of MTOR, including the Ras-Raf-MEK-ERK pathway. ${ }^{45}$ Taken together, our results indicate that the activation of AKT1 signaling pathway in intrahepatic cholangiocarcinoma may occur via alternative pathways that are independent of PTEN, yet the conventional model of the AKT1-MTOR-RPS6KB2/ EIF4EBP1 pathway, except for the upstream effect of PTEN, appears to be valid in this type of tumor.

The treatment of intrahepatic cholangiocarcinomas is challenging because of the aggressive nature of the disease, critical location of the tumor, and lack of effective adjuvant treatment modalities. Complete surgical resection is currently the mainstay of treatment, and it is the only therapeutic option with a chance of cure for patients with intrahepatic cholangiocarcinoma. However, only a limited number of patients with early-stage disease are eligible for curative surgery. The palliative treatments that mainly consist of systemic chemotherapy are of limited benefit as cholangiocarcinomas respond poorly to the existing therapies. ${ }^{46}$ Therefore, further clinical and preclinical trials are necessary to develop novel therapeutic options based on novel targets and tumor markers. In addition, it is becoming increasingly clear that different therapeutic approaches for intrahepatic and extrahepatic cholangiocarcinoma may be necessary, because the intra- and extrahepatic bile ducts have different embryological origins.

In summary, the aberrant expressions of p-AKT1 and p-MTOR in intrahepatic cholangiocarcinoma were associated with a favorable prognosis, possibly through its downstream signaling proteins (p-RPS6KB2 or p-EIF4EBP1), in a PTEN-independent manner. We also demonstrated that PTEN overexpression was an independent favorable prog- nostic factor for patients with intrahepatic cholangiocarcinoma. In addition, the overexpression of p-MTOR was more frequently observed in well-tomoderately differentiated tumors, and in tumors without metastasis. These data may indicate that dysregulation of the AKT1 pathway may have an important role in the development of intrahepatic cholangiocarcinoma, but not necessarily during the progression of the disease, and that the AKT1 pathway may be regulated by an alternative pathway, independent of PTEN in intrahepatic cholangiocarcinoma. Therapeutic strategies targeting AKT1 signaling pathway in intrahepatic cholangiocarcinoma warrants further investigation in light of these new findings.

\section{Acknowledgement}

We thank Mr Hyunsub Lee for his technical assistance.

\section{Disclosure/conflict of interest}

The authors declare no conflict of interest.

\section{References}

1 Nakeeb A, Pitt HA, Sohn TA, et al. Cholangiocarcinoma. A spectrum of intrahepatic, perihilar, and distal tumors. Ann Surg 1996;224:463-473.

2 Patel T. Increasing incidence and mortality of primary intrahepatic cholangiocarcinoma in the United States. Hepatology 2001;33:1353-1357.

3 West J, Wood H, Logan RF, et al. Trends in the incidence of primary liver and biliary tract cancers in England and Wales 1971-2001. Br J Cancer 2006; 94:1751-1758.

4 Jarnagin WR, Shoup M. Surgical management of cholangiocarcinoma. Semin Liver Dis 2004;24:189-199.

5 Altomare DA, Testa JR. Perturbations of the AKT signaling pathway in human cancer. Oncogene 2005;24:7455-7464.

6 Hennessy BT, Smith DL, Ram PT, et al. Exploiting the PI3K/AKT pathway for cancer drug discovery. Nat Rev Drug Discov 2005;4:988-1004.

7 Ermoian RP, Furniss CS, Lamborn KR, et al. Dysregulation of PTEN and protein kinase B is associated with glioma histology and patient survival. Clin Cancer Res 2002;8:1100-1106.

8 Ruggeri BA, Huang L, Wood M, et al. Amplification and overexpression of the AKT2 oncogene in a subset of human pancreatic ductal adenocarcinomas. Mol Carcinog 1998;21:81-86.

9 Staal SP. Molecular cloning of the akt oncogene and its human homologues AKT1 and AKT2: amplification of AKT1 in a primary human gastric adenocarcinoma. Proc Natl Acad Sci USA 1987;84:5034-5037.

10 Sun H, Lesche R, Li DM, et al. PTEN modulates cell cycle progression and cell survival by regulating phosphatidylinositol 3,4,5,-trisphosphate and Akt/ protein kinase B signaling pathway. Proc Natl Acad Sci USA 1999;96:6199-6204. 
11 Jiang BH, Liu LZ. PI3K/PTEN signaling in tumorigenesis and angiogenesis. Biochim Biophys Acta 2008;1784:150-158.

12 Salmena L, Carracedo A, Pandolfi PP. Tenets of PTEN tumor suppression. Cell 2008;133:403-414.

13 Steck PA, Pershouse MA, Jasser SA, et al. Identification of a candidate tumour suppressor gene, MMAC1, at chromosome 10q23.3 that is mutated in multiple advanced cancers. Nat Genet 1997;15:356-362.

14 Yoshimoto M, Cunha IW, Coudry RA, et al. FISH analysis of 107 prostate cancers shows that PTEN genomic deletion is associated with poor clinical outcome. Br J Cancer 2007;97:678-685.

15 Harima Y, Sawada S, Nagata K, et al. Mutation of the PTEN gene in advanced cervical cancer correlated with tumor progression and poor outcome after radiotherapy. Int J Oncol 2001;18:493-497.

16 Hay N, Sonenberg N. Upstream and downstream of mTOR. Genes Dev 2004;18:1926-1945.

17 Sabatini DM. mTOR and cancer: insights into a complex relationship. Nat Rev Cancer 2006;6:729-734 .

18 Shaw RJ, Cantley LC. Ras, PI(3)K and mTOR signalling controls tumour cell growth. Nature 2006;441:424-430.

19 De Benedetti A, Harris AL. eIF4E expression in tumors: its possible role in progression of malignancies. Int J Biochem Cell Biol 1999;31:59-72.

20 Chiang GG, Abraham RT. Targeting the mTOR signaling network in cancer. Trends Mol Med 2007;13:433-442.

21 Hamilton SR, Aaltonen LA (Eds.) World Health Organization Classification of Tumours. Pathology and Genetics of Tumours of the Digestive System. IARC Press: Lyon, 2000.

22 Herberger B, Puhalla H, Lehnert M, et al. Activated mammalian target of rapamycin is an adverse prognostic factor in patients with biliary tract adenocarcinoma. Clin Cancer Res 2007;13:4795-4799.

23 Yoshizawa A, Fukuoka J, Shimizu S, et al. Overexpression of phospho-eIF4E is associated with survival through AKT pathway in non-small cell lung cancer. Clin Cancer Res 2010;16:240-248.

24 Perez-Tenorio G, Stal O. Activation of $\mathrm{AKT} / \mathrm{PKB}$ in breast cancer predicts a worse outcome among endocrine treated patients. Br J Cancer 2002;86:540-545.

25 Chung JY, Hong SM, Choi BY, et al. The expression of phospho-AKT, phospho-mTOR, and PTEN in extrahepatic cholangiocarcinoma. Clin Cancer Res 2009;15:660-667.

26 Gupta AK, Bakanauskas VJ, Cerniglia GJ, et al. The Ras radiation resistance pathway. Cancer Res 2001;61: 4278-4282.

27 Tanno S, Yanagawa N, Habiro A, et al. Serine/ threonine kinase AKT is frequently activated in human bile duct cancer and is associated with increased radioresistance. Cancer Res 2004;64:3486-3490.

28 Javle MM, Yu J, Khoury $\mathrm{T}$, et al. Akt expression may predict favorable prognosis in cholangiocarcinoma. J Gastroenterol Hepatol 2006;21:1744-1751.

29 Schmitz KJ, Lang H, Wohlschlaeger J, et al. AKT and ERK1/2 signaling in intrahepatic cholangiocarcinoma. World J Gastroenterol 2007;13:6470-6477.

30 Lawlor MA, Rotwein P. Insulin-like growth factormediated muscle cell survival: central roles for Akt and cyclin-dependent kinase inhibitor p21. Mol Cell Biol 2000;20:8983-8995.
31 Nogueira V, Park Y, Chen CC, et al. Akt determines replicative senescence and oxidative or oncogenic premature senescence and sensitizes cells to oxidative apoptosis. Cancer Cell 2008;14:458-470.

32 Lu B, Wang L, Stehlik C, et al. Phosphatidylinositol 3-kinase/Akt positively regulates Fas (CD95)-mediated apoptosis in epidermal Cl41 cells. J Immunol 2006;176:6785-6793.

33 Kopnin PB, Agapova LS, Kopnin BP, et al. Repression of sestrin family genes contributes to oncogenic Ras-induced reactive oxygen species up-regulation and genetic instability. Cancer Res 2007;67:4671-4678.

34 Tanaka H, Matsumura I, Ezoe S, et al. E2F1 and c-Myc potentiate apoptosis through inhibition of NF-kappaB activity that facilitates MnSOD-mediated ROS elimination. Mol Cell 2002;9:1017-1029.

35 Kodama Y, Baxter RC, Martin JL. Insulin-like growth factor-I inhibits cell growth in the a549 non-small lung cancer cell line. Am J Respir Cell Mol Biol 2002;27:336-344.

36 Lin B, Kolluri SK, Lin F, et al. Conversion of Bcl-2 from protector to killer by interaction with nuclear orphan receptor Nur77/TR3. Cell 2004;116:527-540.

37 Aki T, Yamaguchi K, Fujimiya T, et al. Phosphoinositide 3-kinase accelerates autophagic cell death during glucose deprivation in the rat cardiomyocyte-derived cell line H9c2. Oncogene 2003;22:8529-8535.

38 Anagnostou VK, Bepler G, Syrigos KN, et al. High expression of mammalian target of rapamycin is associated with better outcome for patients with early stage lung adenocarcinoma. Clin Cancer Res 2009;15: 4157-4164.

39 Xiao L, Wang YC, Li WS, et al. The role of mTOR and phospho-p70S6K in pathogenesis and progression of gastric carcinomas: an immunohistochemical study on tissue microarray. J Exp Clin Cancer Res 2009;28:152.

40 Shah A, Swain WA, Richardson D, et al. Phospho-akt expression is associated with a favorable outcome in nonsmall cell lung cancer. Clin Cancer Res 2005;11:2930-2936.

41 Cappuzzo F, Magrini E, Ceresoli GL, et al. Akt phosphorylation and gefitinib efficacy in patients with advanced non-small-cell lung cancer. J Natl Cancer Inst 2004;96:1133-1141.

42 Lee SH, Kim HS, Park WS, et al. Non-small cell lung cancers frequently express phosphorylated Akt; an immunohistochemical study. APMIS 2002;110:587-592.

43 Mori N, Kyo S, Sakaguchi J, et al. Concomitant activation of AKT with extracellular-regulated kinase 1/2 occurs independently of PTEN or PIK3CA mutations in endometrial cancer and may be associated with favorable prognosiss. Cancer Sci 2007;98:1881-1188.

44 Panigrahi AR, Pinder SE, Chan SY, et al. The role of PTEN and its signalling pathways, including AKT, in breast cancer; an assessment of relationships with other prognostic factors and with outcome. J Pathol 2004;204:93-100.

45 Scheper GC, Morrice NA, Kleijn M, et al. The mitogenactivated protein kinase signal-integrating kinase Mnk2 is a eukaryotic initiation factor $4 \mathrm{E}$ kinase with high levels of basal activity in mammalian cells. Mol Cell Biol 2001;21:743-754.

46 Shimoda M, Kubota K. Multi-disciplinary treatment for cholangiocellular carcinoma. World J Gastroenterol 2007;13:1500-1504.

Supplementary Information accompanies the paper on Modern Pathology website (http://www.nature.com/ modpathol) 